\title{
Validated spectrofluorometric method for determination of gemfibrozil in self nanoemulsifying drug delivery systems (SNEDDS)
}

Ana M. Sierra Villara, Ana C. Calpena Campmanyb, Lyda Halbaut Bellowaa, Monserrat Aróztegui Trenchsa,Beatriz Clares Naverosc

a Pharmacy and Pharmaceutical Technology Department, Faculty of Pharmacy, University of Barcelona, Joan XXIII Avenue, 08028 Barcelona, Spain

b Biopharmacy and Pharmacokinetics Unit, Faculty of Pharmacy, University of Barcelona, Joan XXIII Avenue, 08028 Barcelona, Spain c Pharmacy and Pharmaceutical Technology Department, Faculty of Pharmacy, University of Granada, Campus of Cartuja s/n, 18071 Granada, Spain

\section{HIGHLIGHTS}

- A spectrofluorometric method has been developed for the determination of gemfibrozil.

- The method is based on the excitation and emission capacities of gemfibrozil.

- This method allows de determination of the drug in SNEDDS.

- Results showed linear relationships, low limits of LOD and LOQ with good robustness.

\section{GRAPHICAL ABSTRACT}

Validated spectofluorometric method for determination of gemfibrozil in self nanoemulsifying drug delivery systems (SNEDDS)

Due to the SNEDDS excipient interferences, Gemfibrozil determination was performed by means of a validated espectorfluorometric method, which exhibited satisfactory results allowing release studies to be performed and

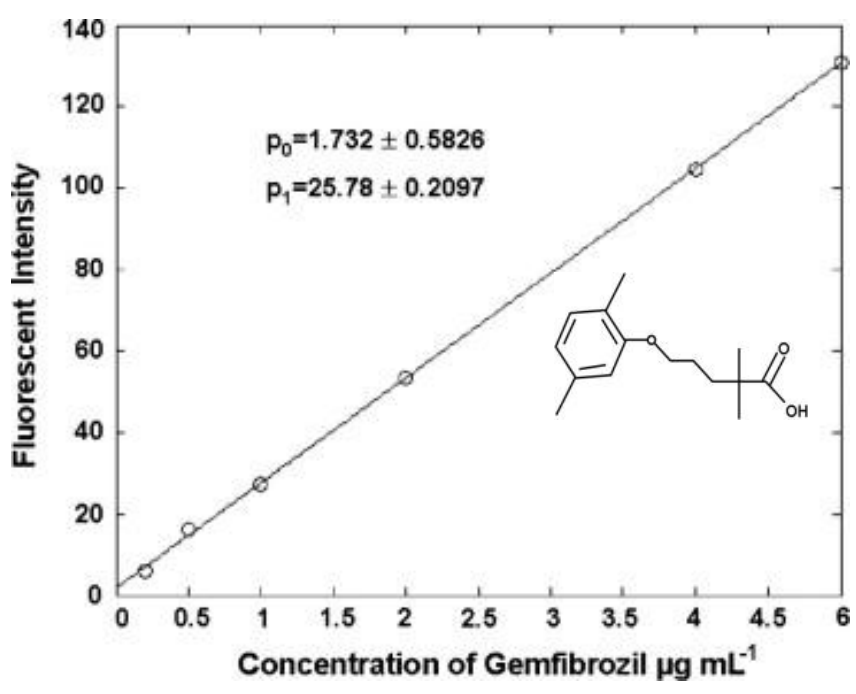

\section{Article history:}

Received 10 December 2012

Received in revised form 19 April 2013

Accepted 24 April 2013

Available online 3 May 2013

Keywords: Gemfibrozil, Fluorescence, Self-nanoemulsifying drug delivery systems, SNEDDS, Spectrofluorometry 


\section{ABSTRACT}

A spectrofluorometric method has been developed and validated for the determination of gemfibrozil. The method is based on the excitation and emission capacities of gemfibrozil with excitation and emission wavelengths of 276 and $304 \mathrm{~nm}$ respectively. This method allows de determination of the drug in a selfnanoemulsifying drug delivery system (SNEDDS) for improve its intestinal absorption. Results obtained showed linear relationships with good correlation coefficients $(r>0.999)$ and low limits of detection and quantification (LOD of $0.075 \mu \mathrm{g} \mathrm{mL}^{-1}$ and LOQ of $0.226 \mu \mathrm{g} \mathrm{mL}^{-1}$ ) in the range of $0.2-5 \mu \mathrm{g} \mathrm{mL}^{-1}$, equally this method showed a good robustness and stability. Thus the amounts of gemfibrozil released from SNEDDS contained in gastro resistant hard gelatine capsules were analysed, and release studies could be performed satisfactorily.

\section{Introduction}

Gemfibrozil (Gem), 5-(2,5-dimethylphenoxy)-2,2-dimethylpentanoic acid, is a benzene derivative of valeric acid (chemical structure provided in the supplementary data) belonging to a drug group known as fibrates. It has been the clinical choice for the hyperlipidemia (type III) and hypertriglyceridemia (type IV) [1] and it has been found to decrease serum triglycerides and very low density lipoprotein-cholesterol and to increase high density lipoprotein-cholesterol [2] by activating the peroxisome proliferator activated receptors (PPARs), acting mainly on the PPAR $\alpha$ isoform [3,4].

Due to its physicochemical properties Gem is a small molecule with poor water solubility, around $0.01 \mathrm{mg} \mathrm{mL}$ [5] and low dissolution rate in the gastrointestinal tract, which limits its effective absorption and bioavailability after oral administration. Thus, it can be assumed that the low oral bioavailability of Gem is due to its solubility and dissolution limitations [5]. Currently, Gem pharmaceutical forms found in the market are tablets and capsules.

In our previous study [6], a self-nanoemulsifying drug delivery system (SNEDDS) was used to conquer the challenge of improving the rate and extent of absorption of Gem, in which the active was loaded in the oily phase. These Gem SNEDDS exhibited a lot of difficulties to be analysed through the most reported technique, high performance liquid chromatography (HPLC) with ultraviolet detection at $276 \mathrm{~nm}[\underline{7}$, 8]due to the excipients included in the formulation. SNEDDS are isotropic mixtures of drug, lipids and surfactants, usually with one or more hydrophilic co-solvents or co-emulsifiers that form fine oil in water nanoemulsions upon mild agitation in an aqueous medium with a droplet sizes ranging 20-200 nm [9]. Different bulk proportions of the mixture components and their physical properties (such as particle size) and the use of different ingredients (such as different excipients) produce changes in the signals from the active ingredients [10]. Spectrofluorometry has long been applied in the field of pharmaceutical analysis of many drugs, being necessary condition for a compound to fluoresce that it absorbs light in the UV or visible region of the spectrum. Most of the additives or excipients found in pharmaceutical preparations are not fluorescent in nature, but compounds that have a conjugated $\pi$-electron system may give efficient reemission of the absorbed energy. In this context, the fluorescence characteristics of Gem let the use of spectrofluorometry as detection technique. This is a technique based on the measurement of the relative fluorescent intensity and the wavelength distribution of fluorescent light emitted from an excited molecule through the absorption of radiant energy [11] providing an attractive methodology because of sensitivity, speed, and simplicity, having a low cost and allows the determination of Gem with enough reliability.

The United States Pharmacopoeia (USP) [12], and the International Conference on Harmonization $(\mathrm{ICH})$ [13]provide a clear description of analytical procedures which govern the validation parameters, that 
need to be evaluated. These parameters are: accuracy, precision (repeatability, intermediate precision), specificity, detection limit, quantification limit, linearity and range.

Therefore, the objective of this work was to develop an accurate, specific and reproducible spectrofluorometric method for the determination of Gem in SNEDDS pharmaceutical dosage forms under study with good sensitivity, relatively simple sample preparation and short run times.

\section{Materials and methods}

\section{Reagents}

Gemfibrozil of analytical reagent grade was purchased from Sigma-Aldrich (Madrid, Spain), methanol and glacial acetic acid (both of HPLC grade) were purchased from Panreac Química S.A.U. (Barcelona, Spain). For the SNEDDS elaboration: Gem was kindly provided by Menarini Lab., S.A. (Badalona, Spain); oils: Lemon essential oil, anise essential oil, peppermint essential oil, soybean oil, as well as, polyoxy 35 castor oil (Cremophor ${ }^{\circledR}$ EL), were purchased from Fagron Iberica S.A.U. (Terrassa, Spain). Mono/diglycerides of caprylic acid (Capmul® MCM-C8) was kindly supplied by Abitec Corp. (Jamesville, USA). Lauroyl macrogol-6 glycerides EP/lauroyl polyoxyl-6 glycerides NF (Labrafil ${ }^{\circ}$ M2130CS) from Gattefossé (Saint-Priest Cedex, France). Potassium dihydrogen phosphate, sodium hydroxide $0.2 \mathrm{M}$, used to prepare buffer solution were purchased from Panreac Química S.A.U. (Barcelona, Spain). Ultra pure water used to prepare all the aqueous solutions was obtained from a Milli- $Q \circledR$ Gradinet $A 10$ system apparatus (Millipore Iberica S.A.U., Madrid, Spain).

\section{Apparatus}

Fluorescence spectra and measurements were made on a SFM 25 Kontron spectrofluorimeter (Kontron Instruments, Basel, Switzerland) equipped with a double beam optical system, wavelength range of 200$800 \mathrm{~nm}$, wavelength accuracy of $1.0 \mathrm{~nm}$ and wavelength precision of $0.1 \mathrm{~nm}$. For the measurements $1.0 \mathrm{~cm}$ quartz cells were utilized and a xenon lamp, with excitation and emission wavelengths of 276 and $304 \mathrm{~nm}$ respectively. The analysis was carried out at ambient temperature $\left(23 \pm 1^{\circ} \mathrm{C}\right)$. The target was just a mobile phase (solution mixture of methanol of analytical reagent grade and glacial acetic acid of analytical reagent grade $1 \% \mathrm{v} / \mathrm{v}$ ) for the calibration curve and a mobile phase plus excipients for the analysis of the samples. The voltage was $320 \mathrm{~V}$. The instrument was calibrated before of this study with good results.

\section{Sample preparation}

The Gem loaded SNEDDS formulations were prepared as follows, accurately weighed Gem was mixed with lemon essential oil in a clear screw thread glass vial. Then, the vial was placed into a water bath using open bath circulators MP-5 (Julabo Labortechnik $\mathrm{GmbH}$; Seelbach, Germany) at $40{ }^{\circ} \mathrm{C}$ for 20 min with gentle stirring to melt the oily mixture and facilitate the solubilization of Gem. Cremophor $\circledast$ EL and Capmul $\circledast$ MCMC8 were accurately weighed and added to the oily mixture using a positive displacement pipette. All weighs were measured using PJ 360 Delta Range analytical balance (Mettler-Toledo; Barcelona, Spain). Formulations were stirred gently using a magnetic stir bar and a magnetic stir plate MELB1719 (Merck Eurolab; Lutterworth, UK) until reaching homogeneous solution. The resulting Gem SNEDDS formulations under study contained Lemon essential oil as oily phase, Cremophor® EL as surfactant and Capmul® MCMC8 as co-surfactant. Loading amount of Gem was $300 \mathrm{mg}$ (F1). Equally, no Gem loaded SNEDDS were 
elaborated (placebo). The composition of developed formulations is shown in Table 1. The method was based on previous studies of this research group [6].

Table 1.

Composition of gemfibrozil SNEEDS formulations utilized for the spectrofluorometric method study.

\begin{tabular}{lllllll} 
Composition & Placebo (mg) & \multicolumn{2}{l}{ Formulations } & & \\
\cline { 3 - 6 } & & F1 (mg) & F80 (mg) & F100 (mg) & F120 (mg) \\
\hline Gemfibrozil & - & 300 & 540 & 600 & 660 \\
\hline Lemon essential oil & 944 & 944 & 944 & 944 & 944 \\
\hline Cremophor® EL & 840 & 840 & 840 & 840 & 840 \\
\hline Capmul@ MCM-C8 & 944 & 944 & 944 & 944 & 944
\end{tabular}

\section{Preparation of reference standard solutions for the calibration curve}

Working standard solutions for the calibration curves were prepared daily as follows; $10 \mathrm{mg}$ of reference standard Gem was accurately weighed and transferred to a $100 \mathrm{~mL}$ volumetric flask. Then it was dissolved in a glacial acetic acid $1 \%(\mathrm{v} / \mathrm{v})$ methanol solution to obtain a final Gem concentration of $100 \mu \mathrm{g} \mathrm{mL}^{-1}$. From this solution, standard stocks of $5,4,2,1,0.5$ and $0.2 \mu \mathrm{g} \mathrm{mL}-1$ were prepared and thermostated at $25 \pm 0.1^{\circ} \mathrm{C}$ in a temperature controlled bath Jubalo MP-5 (Julabo Labortechnik GmbH, Seelbach, Germany).

\section{Analysis of Gem in SNEDDS}

A quantity of SNEDDS equivalent to a $100 \mathrm{mg}$ of Gem was accurately weighed by precision balance PJ360 DeltaRange (Mettler Toledo, Barcelona, Spain) and placed in a $100 \mathrm{~mL}$ volumetric flask and filled with the mobile phase. The solution was mixed until its complete dissolution. This solution was further diluted to obtain Gem concentration of $2 \mu \mathrm{g} \mathrm{mL}^{-1}$ and thermostated at $25 \pm 0.1^{\circ} \mathrm{C}$ in a temperature controlled bath. Then the fluorescent intensity was measured.

The possible interferences from the excipients in the SNEEDS formulations were evaluated. As a control, equal quantity of SNEEDS formulation containing no Gem (placebo) was prepared similarly and fluorescent intensity measured at $304 \mathrm{~nm}$ after excitation at $276 \mathrm{~nm}$.

\section{Method validation}

Validation of the developed spectrofluorometric method was carried out as per the International Conference on Harmonization guidelines Q2 (R1) [13] and included an evaluation of the following characteristics: linearity, sensitivity, accuracy, precision, robustness and analyte stability.

\section{Linearity and calibration curves}

The linearity of the present method was evaluated by constructing the calibration curve at six concentration levels of $5,4,2,1,0.5$ and $0.2 \mu \mathrm{g} \mathrm{mL}^{-1}$. The calibration curve was validate interday $(n=6)$ by different analysts and developed by plotting the instrument measurements versus the corresponding drug concentration. The least squares fit method was employed to statistically evaluate the results for linearity by a regression line and the corresponding slope, $y$-intercept and coefficient of linear correlation $(r)$. Furthermore, linearity was determined by one-way analysis of variance (ANOVA) test to compare the fluorescence signal versus nominal concentrations of each standard, and differences were considered statistically significant when $p<0.05$ [14]. The least square linear regression analysis and mathematical determinations were performed by the Prism®, V. 3.00 software (GraphPad Software Inc., San Diego, CA, USA). 
Equally relative standard deviation (RSD) of the slope might be used as a mathematical measure of linearity, calculated from the quotient of the standard deviation of the slope $\left(\mathrm{SD}_{\mathrm{sb}}\right)$ and the slope [15]:

$$
\text { Linearity }=1-\mathrm{SD}_{\mathrm{sb}} / \mathrm{Sb} \times 100
$$

\section{Sensitivity}

Sensitivity of the method was determined with respect to limit of detection (LOD) and limit of quantification (LOQ). LOD provides information on minimum level at which the Gem was reliably detected by the analysis of samples with known concentrations of Gem and LOQ, in the same way, establishes the minimum level at which Gem was quantified with acceptable accuracy and precision. Both parameters, LOD and LOQ were determined based on the standard deviation of the response and the slope of the calibration curve using the formula:

$$
\mathrm{LOD} \text { or } \mathrm{LOQ}=k \times \mathrm{SD}_{5 \mathrm{a}} / \mathrm{Sb}
$$

where $k$ is the a factor related to the level of confidence, its value is 3.3 for LOD and 10 for LOQ. SD sa is the standard deviation of the intercept and $\mathrm{Sb}$ is the slope [16].

\section{Accuracy}

Accuracy of the method was performed by the addition technique. In this technique, three different known analytical amounts of Gem corresponding to $80 \%, 100 \%$ and $120 \%$ of the nominal Gem concentration corresponding to 240,300 and $360 \mathrm{mg}$ respectively, were added to the SNEEDS formulation (F1). Then accuracy of the method was evaluated carrying the recovery study at those three levels in six replicates.

\section{Precision: repeatability and intermediate precision}

The precision of the assay was determined by the intraday repeatability. For this, SNEDDS formulations containing Gem at three levels, low, medium and high concentration (F80, F100 and F120; Table 1) were analysed during the same day (while keeping the operating conditions identical) in six replicates. Equally, the intermediate precision (interday) was assessed by analyzing Gem in SNEDDS at these three levels with six replicates of each sample over six days. The data obtained were used to calculate mean and \%RSD (relative $\mathrm{SD})$ values.

\section{Robustness}

Robustness of the procedures was assessed by evaluating the influence of small variation in experimental variables: concentration of excipients, slight change in the glacial acetic acid methanol solution and ambient temperature. The effects on the results were evaluated and robustness determined in triplicate and the mean and $\% R S D$ of responses were calculated.

\section{Stability}

The stability tests were performed by freeze thawing cycle. Three set formulations, F80, F100 and F120 were subjected to 2 freeze-thaw cycles, which included freezing at $-4{ }^{\circ} \mathrm{C}$ for $24 \mathrm{~h}$ followed by thawing at $40{ }^{\circ} \mathrm{C}$ for 24. Gem was subsequently analysed. Equally stock solutions were checked for stability by freezer storage conditions at $-50^{\circ} \mathrm{C}$ for $24 \mathrm{~h}$. 
The samples were considered to be stable at a given condition if the mean values obtained from the treated formulations were within $\pm 10 \%$ of the mean values of the untreated or reference samples that were run within the same analytical run.

\section{Application of the method}

Based on the Box-Behnken design, fifteen Gem-loaded SNEDDS with different concentrations of surfactant, co-surfactant, and oil, but containing Gem at a final loading of $300 \mathrm{mg}$, were filled into size 00 HPMC hard gelatine capsules required for release studies. Then the capsules were coated with Eudragit $₫ L$ by successive layer coating process.

In vitro dissolution tests were performed using a USP XXIV paddle apparatus SR8 PLUS (Hanson Research, Canada) at $37 \pm 0.5^{\circ} \mathrm{C}$ and rotating speed of $50 \mathrm{rpm}$ in $900 \mathrm{~mL}$ of phosphate buffer solution (pH 7.5; $0.05 \mathrm{M}$ ). The coated capsules with SNEDDS containing $300 \mathrm{mg}$ of Gem were held to the bottom of the vessel using copper sinkers. Five $\mathrm{mL}$ aliquots were removed at predetermined time intervals; 1, 3, 5, 10, 15, 30 and $45 \mathrm{~min}$ from the dissolution medium and replaced with fresh buffer and were tested for Gem using the spectrofluorometric method.

Data obtained from the in vitro release studies were measured, as detailed in the following section, and values reported as the mean $\pm S D$ of the six replicates. Four different kinetic models (zero order, first order, Higuchi and Weibull function) were used to fit the experimental data obtained in the drug release experiment[17].

$$
\begin{aligned}
& \% R_{t} / \% R_{\infty}=k \times t \quad \text { Zeroorder } \\
& \% R_{t} / \% R_{\infty}=1-e^{-K \times t} \quad \text { First-order } \\
& \% R_{t} / \% R_{\infty}=k \times t^{1 / 2} \quad \text { Higuchi's equations } \\
& \% R_{t} / \% R_{\infty}=1-e^{-(t / t d) \beta} \quad \text { Weibull's equation }
\end{aligned}
$$

where $\% R_{t}$ is the percentage drug released at time $t, \% R_{x}$ is the total percentage drug released, $\% R_{t} / \% R_{x}$ is the fraction of drug released at time $t, K$ is the release rate constant, $t d$ is the time in which the $63.2 \%$ of the drug is released and $B$ is the shape parameter.

A nonlinear least-squares regression was performed using the WinNonLin® Professional edition version 3.3 software (Pharsight Corporation, USA), and the model parameters calculated. Also the Akaike's Information Criterion (AIC) was determined for each model as it is an indicator of the model's suitability for a given dataset [18]. The smaller the value of AIC, the better the model adjusts the data.

Besides, some additional amodelistic parameters as dissolution efficiency (DE) and mean dissolution time (MDT) were also calculated from the in vitro release data. These parameters are very useful to compare different profiles types because the evaluation of dissolution characteristics of the different formulations is not conditioned by the goodness of the fitting to a theoretical model of our data. The DE (\%) was calculated by mean of the following equation:

$$
\operatorname{DE}(\%)=\frac{\int_{0}^{t} M \times d t}{C_{100} \times t} \times 100
$$


where the numerator represents the area under the dissolution curve up to a certain time, $t$, and the denominator is the rectangular area between the total amount of drug released and the last experimental time point, $t$. For area under dissolution curve (AUC) calculation, a continuous plot of the evolution of released quantities across the whole of the experimental time was calculated. Afterward, respective AUC values were calculated by means of a trapezoidal rule for the whole time values interval.

MDT is defined as the mean residence time of a drug in the formulation. It is a useful parameter to characterize the drug release rate from a dosage form. It was calculated using the following equation:

MDT $=\frac{\sum_{i=1}^{n} \bar{t}_{i} \times \Delta M_{i}}{M_{\infty}}$

where $i$ is the sample number, $n$ is the number of dissolution sample times, $t_{i}$ is the time at midpoint between $t_{i}$ and $t_{i=1}$ (calculated with the expression $\left(t_{i}+t_{i-1}\right) / 2$ ), $\Delta M_{i}$ is the increase in the amount of drug released at each time interval, and $M_{\infty}$ is the asymptote of the dissolved amount of drug. Both amodelistic parameters were reliably calculated because the maximum percentage of drug released was $90 \%$ [19].

\section{Results and discussions}

\section{Validation of the method}

To evaluate the linearity and determine the concentration of the Gem in the SNEDDS formulation under study from Gem standard solutions six calibration curves were constructed under optimal conditions. These curves were useful to know the linearity, range, detection and quantitative limits. It was found that the fluorescence intensity $(F)$ was proportional to that of the concentration of Gem $\left(\mu \mathrm{g} \mathrm{mL}^{-1}\right)$ in the range $5-0.2 \mu \mathrm{g} \mathrm{mL}^{-1}$ as shown in Fig. 1. The representative linear equation was $F=1.732+25.78 C$, where $C$ is Gem concentration (expressed as $\mu \mathrm{g} \mathrm{mL}^{-1}$ ), with a determination coefficient $(r)>0.999$ in all curves. No statistically significant differences were found $(p>0.05)$. The LOD was $0.075 \mu \mathrm{g} \mathrm{mL}^{-1}$ (confidence level of 3.3 for our system) and

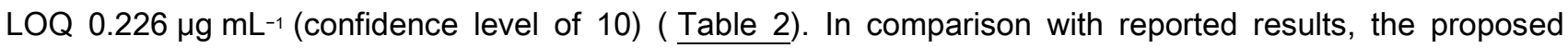
method possesses comparable analytical parameters [20]. Linearity of this method was also determined in terms of RSD to be $98.8 \%$.

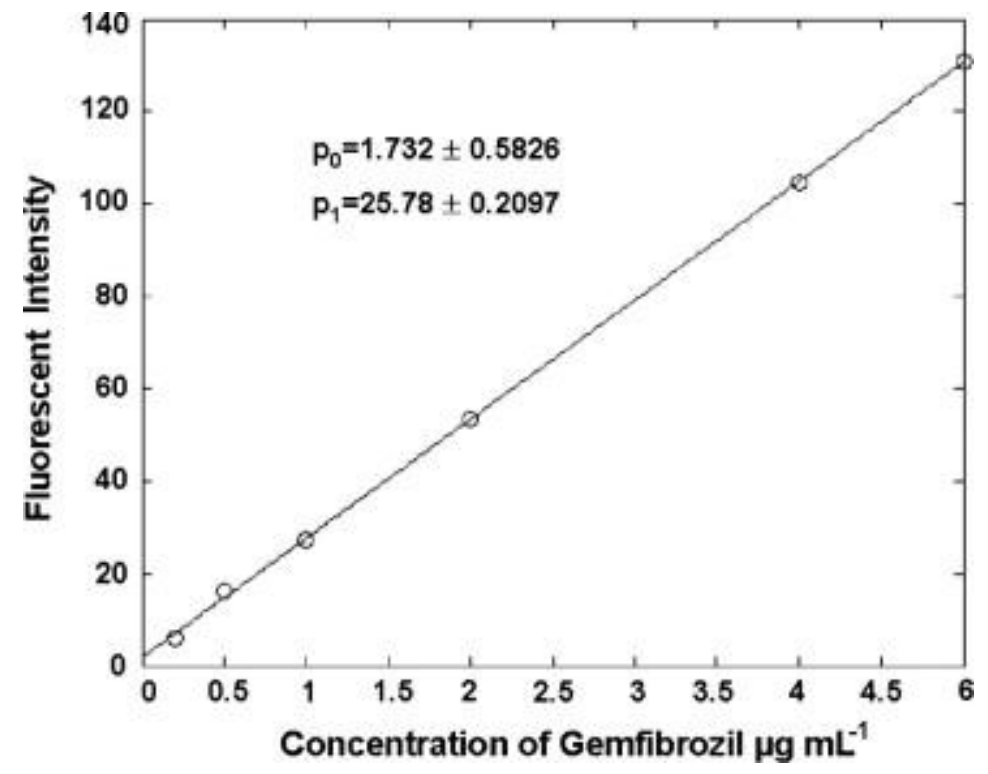

Fig. 1.

Spectrofluorometric calibration curve of gemfibrozil stock solutions. 
Table 2.

Results of the analysis of the data for the method validation parameters for determination of gemfibrozil in SNEDDS.

\begin{tabular}{|l|l|l|}
\hline Statistical parameter & Values & SD $(n=6)$ \\
\hline Concentration range $(\mu / \mathrm{mL})$ & $5-0.2$ & \\
\hline Fit equation & $F=25.78 C+1.732$ & \\
\hline Determination coefficient $\left(r^{2}\right)$ & 0.9997 & 0.0001 \\
\hline Slope $(\mathrm{Sb})$ & 25.78 & 0.297 \\
\hline Intercept $(\mathrm{Sa})$ & 1.732 & 0.583 \\
\hline Limit of detection $(\mathrm{LOD})(\mu / \mathrm{mL})$ & 0.075 & \\
\hline Limit quantification $(\mathrm{LOQ})(\mu / \mathrm{mL})$ & 0.226 & \\
\hline
\end{tabular}

Accuracy is reported in Table 3 as the percentage of recovery by the assay of known added amounts of Gem in the SNEDDS. The overall percent recovery of Gem was $98.46 \%$ and relative standard deviation ranging from $3.28 \%$ to $4.63 \%$.

Table 3.

Validation data for accuracy of the proposed method for analysis of gemfibrozil in SNEDDS.

\begin{tabular}{|l|l|l|l|l|l|l|}
\hline Formulation & Drug content $(\mathrm{mg})$ & Drug added $(\mathrm{mg})$ & Theoretical concentration $(\mathrm{mg})$ & Found $(\mathrm{mg})$ & $\%$ Recovery & RSD $(\%)$ \\
\hline F1 & 300 & 240 & 540 & 537.51 & 99.54 & 3.48 \\
\hline F1 & 300 & 300 & 600 & 583.56 & 97.26 & 4.63 \\
\hline F1 & 300 & 360 & 660 & 650.62 & 98.58 & 3.28 \\
\hline
\end{tabular}

a

Average of six determinations.

The precision of this method was determined by repeatability within-day, using three different concentrations of the SNEDDS of Gem. Every concentration was analysed in six replicates. Also, intermediate precision between days was evaluated using these three different concentrations of Gem. The results obtained are presented in Table 4, values in both cases were less than 3\% (RSD) confirming satisfactory results.

Table 4.

Validation data for precision of the proposed method for analysis of gemfibrozil in SNEDDS.

\begin{tabular}{|c|c|c|c|c|}
\hline \multirow[t]{2}{*}{ Formulation } & \multirow[t]{2}{*}{ Intraday assay } & \multicolumn{2}{|c|}{ Recovery $(n=6)$} & \multirow[t]{2}{*}{$\operatorname{RSD}(\%)$} \\
\hline & & $\operatorname{RSD}(\%)$ & Interday assay & \\
\hline F80 & 96.36 & 1.22 & 96.65 & 1.30 \\
\hline F100 & 99.54 & 2.41 & 99.48 & 2.43 \\
\hline F120 & 97.51 & 2.45 & 96.90 & 2.36 \\
\hline
\end{tabular}

The most attractive feature of the spectrofluorimetric method is its relative freedom from pharmaceutical additives and excipients. In this case the pharmaceutical additives and adjuvants are not fluorescent at the excitation and emission wavelengths used and did not interfere in the determination. The specificity of the fluorescence of the molecules makes difficult any interference, but not impossible. In spite of this, if it will occur, probably only will affect the background fluorescence of the solution, and as the quantification method used is standard addition the problem will be overcomed.

The determination of Gem under the various conditions was analysed by the present method. Robustness shows the reliability of an analyte with respect to deliberate variations in method parameters. Only one parameter was changed in the experiments at a time. The operational parameters challenged to prove the robustness include changes in excipients concentration (Lemon essential oil: Capmul囚 MCM-C8; 1:0.75), glacial acetic acid methanol solution (3\%) and ambient temperature of $25^{\circ} \mathrm{C}$. In these experiments one 
parameter was changed while the other parameters were kept unchanged, and the recovery percentage was calculated (Table 5). The small variations in any of the variables did not significantly affect the results. This gave an indication for the reliability of the proposed method during routine work.

Table 5.

Influence of small variation in the assay conditions on the analytical performance of the proposed method for analysis of gemfibrozil in SNEDDS.

\begin{tabular}{|c|c|c|c|c|}
\hline Parameter & Drug content (mg) & Found $(\mathrm{mg})$ & $\%$ Recovery $^{\mathrm{a}}$ & $\operatorname{RSD}(\%)$ \\
\hline Excipient concentration & 300 & 298.62 & 99.54 & 3.48 \\
\hline Glacial acetic acid methanol solution ( $3 \%)$ & 300 & 291.11 & 97.26 & 4.63 \\
\hline Temperature $25^{\circ} \mathrm{C}$ & 300 & 295.74 & 98.58 & 3.28 \\
\hline
\end{tabular}

a

Each value is the mean of three determinations.

Gem stability was determined under a variety of conditions. As described above, formulations were used to show that the analyte is stable through at least two freeze-thaw cycles. The stock solutions of both were shown to be stable when stored at $-50{ }^{\circ} \mathrm{C}$. During the stability testing, no deterioration of Gem was observed. For each stability test, treated and control samples were within $\pm 10 \%$ of each other and precision $(n=3)$ was within $\pm 15 \%$.

\section{Application of the method}

Fifteen Gem loaded SNEDDS formulation containing $300 \mathrm{mg}$ of Gem were subjected to the analysis by the proposed spectrofluorometric method and the obtained results are shown in Table 6 . The mean percentage recoveries ranged from $97.3 \%$ to $104.1 \%$ at the end of the release study. Exicipients of the formulation did not present fluorescent emission that interferes with the emission of Gem. Therefore, an extraction procedure was not necessary.

Table 6.

Content of gemfibrozil in various SNEEDS formulations.

\begin{tabular}{|l|l|l|l|}
\hline Formulation & Gemfibrozil (mg) & Recovery $\mathbf{~}(\%)$ & RSD $(\%)$ \\
\hline 1 & 300 & 96.8 & 3.82 \\
\hline 2 & 300 & 99.1 & 3.53 \\
\hline 3 & 300 & 103.6 & 5.73 \\
\hline 4 & 300 & 97.3 & 2.87 \\
\hline 5 & 300 & 101.2 & 3.43 \\
\hline 6 & 300 & 99.3 & 3.19 \\
\hline 7 & 300 & 104.1 & 4.48 \\
\hline 8 & 300 & 97.5 & 3.24 \\
\hline 9 & 300 & 98.7 & 2.86 \\
\hline 10 & 300 & 101.9 & 3.79 \\
\hline 11 & 300 & 98.8 & 3.35 \\
\hline 12 & 300 & 99.6 & 3.58 \\
\hline 13 & 300 & 102.3 & 4.77 \\
\hline 14 & 300 & 98.2 & 3.26 \\
\hline 15 & 300 & 97.5 & 3.28 \\
\hline$a$ & & & \\
\hline Each value is the mean of three determinations. & \\
\hline
\end{tabular}


Data obtained from the spectrofluorometric method enabled a more accurate study and understanding of the release profiles by fitting to theoretical kinetics models, as well as, determination of the mean residence time of a drug in the formulation or the dissolution efficiency (amodelistic parameters).

The release profiles indicated a two step process for SNEDDS. The initial step shows a burst release which can be attributed to the surface associated drug, followed by a slower sustained release phase. The phenomenon exhibits that the release of Gem is controlled by diffusion.

The kinetic model that exhibited the smaller value of AIC and higher correlation coefficient of the linear regressions $\left(r^{2}\right)$ thus, statistically described best the drug release mechanism were the Weibull function, with a $Q_{\infty}$ value of 98.6 (\% Gem). On the use of the Weibull function for the discernment of drug release mechanisms, estimates for $\beta \leqslant 0.75$ indicate Fickian diffusion in either fractal or Euclidian spaces while a combined mechanism (Fickian diffusion and swelling controlled release) is associated with $\beta$ values in the range $0.75<\beta<1$. For values of $\beta$ higher than 1 , the drug transport follows a complex release mechanism[21]. For Gem-SNEDDS in coated capsules $\beta$ value is 1.74 . This $\beta$ value has been described as a sigmoid curve indicative of complex release mechanism, the rate of release does not change monotonically. In fact, the release rate initially increases nonlinearly up to the inflection point and thereafter decreases asymptotically [21].

Only for $t_{d}$ values which were 5.29 for Gem-SNEDDS in coated capsules, $t_{d}$ represents the time interval necessary to dissolve or release $63.2 \%$ of the drug present in the pharmaceutical dosage form, meaning that in 45 min study, $63.2 \%$ of Gem was released in 5.29 min from SNEDDS.

Equally amodelistic pharmacokinetic parameters independent of the release model such as EF and MDT were calculated with the experimental values $(n=6)$. It could be observed that $78.3 \% \mathrm{EF}$ value from the coated capsules of SNEDDS. Importantly, the drug dissolution from a dosage form plays an important role in the development of new drug formulations. On the other hand, MDT was $3.1 \pm 0.1 \mathrm{~min}$.

\section{Conclusion}

Gem released from the SNEEDS formulations was satisfactorily determined by using the proposed spectrofluorometric method, direct spectrofluorimetry is a simple procedure to determine Gem. There was no interference from the excipients in Gem detection. The method showed optimal results in linearity, sensitivity, accuracy, precision and robustness and stability.

\section{Acknowledgements}

The authors want to acknowledge Dr. Isidre Casals and his team of the department of Liquid Chromatography of the Scientific-Technical Services of the University of Barcelona for their technical support.

\section{Appendix A. Supplementary material}

Supplementary data associated with this article can be found, in the onlineversion, at http://dx.doi.org/10.1016/.saa.2013.04.092.

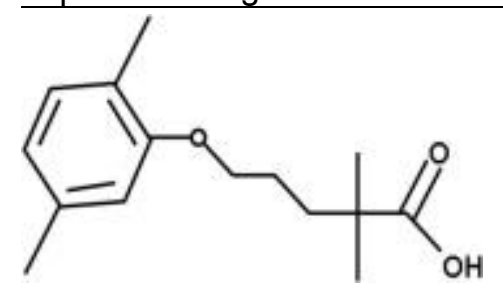

Supplementary Figure 2. 


\section{References}

1. S.C. Sweetman, S. Martindale. The Complete Drug Reference. 34th ed. Pharmaceutical Press, London, 2005.

2. S. Kersten, S. Mandard, N.S. Tan, P. Escher, D. Metzger, P. Chambon, F.J. Gonzalez, B. Desvergne, W. Wahli. J. Biol. Chem., 275 (2000), pp. 28488-28493.

3. R.S. Loomba, R. Arora. Ther. Adv. Cardiovasc. Dis., 3 (2009), pp. 91-96.

4. J.C. Fruchart, P. Duriez. Drugs Today, 42 (2006), pp. 39-64.

5. N.A. Kasim, M. Whitehouse, C. Ramachandran, M. Bermejo, H. Lennernäs, A.S. Hussain, H.E. Junginger, S.A. Stavchansky, K.K. Midha, V.P. Shah, G.L. Amidon. Mol. Pharm., 1 (2004), pp. 85-96.

6. A.M. Villar, B.C. Naveros, A.C. Campmany, M.A. Trenchs, C.B. Rocabert, L.H. Bellowa. Int. J. Pharm., 431 (2012), pp. 161-175

7. Y.M. Chen, P.C. Lin, M. Tang, Y.P. Chen. J. Supercrit. Fluids, 52 (2010), pp. 175-182

8. Q.P. Huang, J.X. Wan, G.Z. Chena, Z.G. Shen, J.F. Chen, J. Yun. Int. J. Pharm., 360 (2008), pp. 58-64.

9. C.J.H. Porter, C.W. Pouton, J.F. Cuine, W.N. Charman. Adv. Drug Deliv. Rev., 60 (2008), pp. 673-691.

10. J.C.L. Alves, R.J. Poppi. Spectrochim. Acta A (2012) http://dx.doi.org/10.1016/j.saa.2012.10.074

11. B. Tang, B. Jia, G. Cui, Y. Ding. Anal. Chim. Acta, 516 (2004), pp. 221-227.

12. USP29-NF24, The United States Pharmacopoeia Convention, Rockville, 2006.

13. ICH, Validation of Analytical Procedures: Text and Methodology Q2 (R1), EMEA, 2006.

14. D.L. Massart, B.G.M. Vandeginste, L.M.C. Buydens, S. De Jong, P.J. Lewi, J. Smeyers-Verbeke. Handbook of Chemometrics and Qualimetrics: Part A. Elsevier, Amsterdam, 1997.

15. L. Cuadros Rodriguez, A.M. Garcia Campaña, J.M. Bosque Sendra. Anal. Lett., 29 (1996), pp. 1231-1239.

16. E. McEvoy, S. Donegan, J. Power, K. Altria. J. Pharm. Biomed. Anal., 44 (2007), pp. 137-143.

17. P. Costa, J.M. Sousa. Eur. J. Pharm. Sci., 13 (2001), pp. 123-133.

18. K. Yamaoka, T. Nakagawa, T. Uno. J. Pharmacokinet. Biopharm., 6 (1978), pp. 165-175.

19. K.A. Khan. J. Pharm. Pharmacol., 27 (1975), pp. 48-49.

20. J.L. Manzoori, M. Amjadi. J. Pharm. Biomed. Anal., 31 (2003), pp. 507-513.

21. V. Papadopoulou, K. Kosmidis, M. Vlachou, P. Macheras. Int. J. Pharm., 309 (2006), pp. 44-50. 